\title{
Three-dimensional analysis of gait in postmenopausal women with low bone mineral density
}

\author{
Abeer M ElDeeb ${ }^{1 *}$ and Amr S Khodair ${ }^{2}$
}

\begin{abstract}
Background: There's lack in the literature respecting changes in the trunk and hip angles, and power profile of the lower extremities in postmenopausal women with low bone mineral density (BMD). Therefore, this study aimed to examine gait characteristics of that population, and find out which characteristics may be predictors to BMD. This may provide suitable interventions for subjects with osteoporosis.

Methods: Seventeen healthy postmenopausal women and seventeen with low BMD engaged in this study. Dual $X$-ray Absorbiometry measured BMD at lumber $\left(L_{2-4}\right)$ and femoral neck. Qualysis gait analysis system assessed the gait pattern of each subject.

Results: Compared to healthy peers, women with low BMD showed less trunk rotation $(p=0.02)$, hip adduction $(p=0.005)$ and extension moments $(p=0.008)$. They showed less hip power generation during early stance (H1S) $(p=0.000)$, and swing phase (H3S) ( $p=0.005)$, and less hip power absorption (H2S) $(p=0.005)$. They also, showed less knee power absorption during terminal swing (K4S) ( $p=0.002)$, and ankle power generation at push off (A2S) $(p=0.000)$. The ability of the gait variables to discriminate between subjects with or without osteopenia was $(0.72 \%$, $p=0.016)$ for trunk rotation, $(78 \%, p=0.0004)$ for hip adductor moment, $(76 \%, p=0.0013)$ for hip extensor moment, $(87 \%, p<0.0001)$ for H1S, $(79 \%, p=0.0001)$ for $\mathrm{H} 2 \mathrm{~S},(77 \%, \mathrm{p}=0.0008) \mathrm{H} 3 \mathrm{~S},(81 \%, \mathrm{p}=0.0001)$ for $\mathrm{K} 4 \mathrm{~S}$, and $(93 \%, p<0.0001)$ for A2S.
\end{abstract}

Conclusion: Less power generation at the hip and ankle as well as, less power absorption at the hip and knee, may suggest that postmenopausal women with low BMD showed less propulsion, and stability during walking. Trunk rotation, hip adduction and extension moments, H1S, H2S, H3S, K4S, and A2S are significant predictors for low bone mass in the postmenopausal women.

Keywords: Gait, Moment, Power, Bone mineral density, Menopause

\section{Introduction}

Osteoporosis is a major, public, healthy problem, which increases proportionally according to age. It is a skeletal disorder compromising bone strength, and predisposing the subject to an increased risk of fractures in the hip, spine, and other sites [1].

Women after menopause are more susceptible to osteoporosis than men due to hypoestrogenism [2]. They

\footnotetext{
*Correspondence: beroeldeeb@yahoo.com

'Department of Physical Therapy for Women's Health, Faculty of Physical

Therapy, Cairo University, Cairo, Egypt

Full list of author information is available at the end of the article
}

showed changes in muscle strength and mass [3], which affect gait [4], independence, and quality of life [5].

Gait studies give a clear understanding of demands placed on the musculoskeletal and neurological systems in normal subjects and patients [6]. Few studies have explored the changes in the gait of the postmenopausal women. Women with low bone mineral density (BMD) walk with slow gait speed and large step time and stance time [7], which associated with BMD at the hip, spine, and forearm [8]. The previous studies have focused on examining the spatio-temporal parameters of gait. These parameters are only results of complicated motor pattern 
that do not describe the gait pattern or distinguish between gait changes or their compensations [9].

Some studies have proved that joint moments during gait affect BMD. These studies have reported significant relations between joint moments and BMD in normal subjects [10], elderly [11], and patients with hip [12] or knee osteoarthritis [13]. The previous results lead to hypothesize that gait parameters in postmenopausal women with low BMD may be different from their healthy controls. These gait parameters may be associated with BMD.

So, the first objective of this study was to compare trunk and hip kinematics, joint moments, and power profile between postmenopausal women with low BMD and healthy controls. The second objective was to find out which gait characteristics related to BMD.

\section{Methods}

\section{Subjects}

The database of Dual X-ray Absorbiometry (DEXA) computer, at Nasser Institute Hospital for Research and Therapy, was searched in the preceding year. Postmenopausal women with normal and low BMD were contacted, and asked to engage in this study. A doctor assessed them for inclusive and exclusive criteria of the study. They should be 50-65 years old; independent; sedentary; had a natural menopause for $\geq 3$ years, and they did not engage in any exercise program or sport training. Women were excluded if they had a history of spondylolisthesis, diabetes, hypertension, cardiopulmonary, neuromuscular and renal diseases, thrombosis, back/leg deformities or surgeries, oophorectomy, osteoarthritis and hormonal replacement therapy.

Subjects were assigned into two groups based on WHO definitions of T-score of BMD. They were assigned to normal BMD group when T-score "at one or more skeletal sites" was within 1 standard deviations of the mean for healthy adults. Subjects, with T-score between -1.0 and -2.5 standard deviation of the mean for healthy adults, were assigned to low BMD group. Full instructions about the assessment procedures had been given to each subject, who had signed a consent form. The Ethical Committee of Faculty of Physical Therapy, Cairo University, Egypt, approved the protocol of this study.

Ten normal women and nine with low BMD completed the assessment procedures. Their results were used to decide the sample size. There was a significant difference between them in the hip extension moment, which showed a medium effect size (cohen's $d=1.007$, $r=0.45$ ). A sample of 17 women per group should be recruited to detect a medium size effect of $r=0.45$ at a power of 0.80 , and alpha level of 0.05 .

T-score of the normal BMD group ranged from -0.11 to -1 at $\mathrm{L}_{2-4}$, and -0.89 to -1 at the femoral neck. For low BMD group, it ranged from -1.23 to -2.45 at the $\mathrm{L}_{2-4}$, and -1.2 to -2.45 at the femoral neck. Not all postmenopausal women with low BMD received $\mathrm{Ca}$ and vitamin $\mathrm{D}$ supplements.

\section{Anthropometric and BMD measurements}

A full history of each subject was collected at starting of this study. Weight-height scale was used to measure weight and height for all subjects. The scale was calibrated; each subject stood two times and the average of the weight was recorded. Body mass index (BMI) was calculated according to the formula; weight divided by height square $(\mathrm{Kg} / \mathrm{m} 2)$. DEXA (Unigramm X-ray Plus; IIb (93/42/CEE); UPG-00-174/01; Italy), measured BMD (gram/cm2) at $\mathrm{L}_{2-4}$ and femoral neck. Although almost subjects had DEXA scan since 6 months, BMD was measured again to gain the most accurate recent data of the subjects.

\section{Data collection and processing}

Qualisys gait system (Qualisys Medical AB, Gothenburg, Sweden) was used to analyze the gait pattern of each subject. Qualisys provided a valid and reliable data $[14,15]$. It consisted of six cameras, a force plate, and a personnel computer (pc). The cameras, (type170120; 100-240 V; 50-60 HZ; $230 \mathrm{~mA}$ ), had a capture rate of 120 frames per second. They located at suitable positions, from 10-meter walkway, to view the measurement volume. They synchronized with an $\mathrm{AMTI}^{\mathrm{b}}$ kistler force plate (with external amplifier 9865) located in the middle of the walkway. The pc was installed with Q Trac and Q Gait software. The pc had a Microsoft window 98, 2nd ed., 4.10.2222 A; registered to Medical Eng. System Co.(16201-OEM- 0094512-06975); and supported by a crest computer (BE, Genuine lntel, $\mathrm{X}$ 86 Family 6, Model 8 stepping 6, 127.0 MBRAM).

The therapist started the gait analysis by calibrating the cameras. She placed shoulder, sacrum, and feet markers on each subject, who stood in the middle of the walkway. She adjusted Q trac at the calibrating mode; at least two cameras should pick up each marker. Then, she calibrated the measurement volume by using a wand kit (type 130440). Lshape wand was placed on the force plate with the $\mathrm{X}$-axis in the walking direction. Then, T-shape wand was moved in $\mathrm{X}, \mathrm{Y}$, and $\mathrm{Z}$ directions to allow all cameras to pick up the markers positions in various locations. Force plate position was calibrated by placing four markers at its corners. Then, data was captured, tracked, and exported.

Twenty reflecting markers were placed on special bony landmarks of each subject according to the motion system software. They were placed as follows: the tip of each acromion, spinous process of $12^{\text {th }}$ thoracic vertebra, and the sacrum (between the right and left posterior superior iliac spine). Other markers were placed on the anterior superior iliac spine, greater trochanter, lateral 
side of the knee joint center, superior surface of the patella, tibial tuberosity, lateral malleolus, heel, and between the distal ends of the $2^{\text {nd }}$ and $3^{\text {rd }}$ metatarsals of each leg.

Then, each subject walked along the walkway three trials at her self-selected speed without knowing the presence of the force plate to step on it without targeting. The three-dimensional coordinates of the markers were calculated and filtered by Q Trac and Q Gait software. The collected data were: trunk tilt, rotation and obliquity range of motion (ROM), hip sagittal, coronal and transverse ROM, hip extension moment at midstance, adduction moment at loading response, and internal and external rotation moments. Power profile of the lower extremities in the sagittal plane was also, collected. According to Eng and Winter [16], the work done by the muscles was represented by two capital letters and one number. The first capital letter points out the joint $(\mathrm{H}=$ hip, $\mathrm{K}=$ knee, $\mathrm{A}=$ Ankle); the number points out the power burst position; and the last capital letter points out the plane ( $\mathrm{S}=$ sagittal).

\section{Statistical analysis}

Mann-Whitney $U$-test was used to compare between two groups. Spearman's correlation coefficients assessed the relationship between BMD and gait variables. SPSS version 13.0 (SPSS Inc., Chicago, IL, USA) carried out analysis of the data. Receiver operating curve (ROC) assesses discriminative properties of the gait variables. MedCalc (version 13.0.2) calculated area under curve (AUC), sensitivity, specificity; positive predictive value $(\mathrm{PPV})$ and negative predictive value (NPV). The probability value was considered significant at $<0.05$.

\section{Results}

\section{Subjects' characteristics}

No significant difference was found between subjects' characteristics including age, height, and postmenopausal years. However, low BMD group had lower BMI $(\mathrm{p}=0.01)$ than normal group (Table 1).

\section{Kinematics and kinetics parameters}

Tables 2 and 3 represent kinematics and kinetics parameters of normal and low BMD groups. Results showed no

Table 1 Subjects' characteristics

\begin{tabular}{llll}
\hline Variable & $\begin{array}{l}\text { Normal BMD } \\
\text { group }(\mathbf{N}=\mathbf{1 7})\end{array}$ & $\begin{array}{l}\text { Low BMD group } \\
(\mathbf{N}=\mathbf{1 7})\end{array}$ & $\begin{array}{l}\text { MannWhitney } \\
\text { p-value }\end{array}$ \\
\hline Age $(\mathrm{yrs})$ & $53.23 \pm 4.52$ & $55.88 \pm 4.94$ & 0.09 \\
Height $(\mathrm{m})$ & $1.57 \pm 0.04$ & $1.54 \pm 0.05$ & 0.10 \\
BMl $\left(\mathrm{Kg} / \mathrm{m}^{2}\right)$ & $29.91 \pm 1.91$ & $28.59 \pm 1.53$ & $0.01^{*}$ \\
Postmenopausal yrs & $4.94 \pm 2.81$ & $5.64 \pm 2.91$ & 0.36 \\
\hline
\end{tabular}

Values are mean $\pm S D, B M D$ : bone mineral density, $p$ : probability, ${ }^{*} \mathrm{p}<0.05$.
Table 2 Kinematics parameters of the postmenopausal women

\begin{tabular}{llll}
\hline Variable & $\begin{array}{l}\text { Normal BMD } \\
\text { group }(\mathbf{N}=\mathbf{1 7})\end{array}$ & $\begin{array}{l}\text { Low BMD group } \\
(\mathbf{N}=\mathbf{1 7})\end{array}$ & $\begin{array}{l}\text { MannWhitney } \\
\mathbf{p} \text {-value }\end{array}$ \\
\hline Trunk & & & \\
Tilt ROM & $4.05 \pm 1.31$ & $4.34 \pm 1.79$ & 0.53 \\
Rotation ROM & $16.06 \pm 4.46$ & $12.60 \pm 4.96$ & $0.02^{*}$ \\
Obliquity ROM & $9.49 \pm 2.59$ & $8.52 \pm 3.03$ & 0.32 \\
Hip & & & \\
Sagittal ROM & $41.24 \pm 7.36$ & $38.39 \pm 5.57$ & 0.17 \\
Coronal ROM & $11.50 \pm 3.68$ & $11.42 \pm 3.17$ & 0.86 \\
Transverse ROM & $14.28 \pm 2.36$ & $12.54 \pm 3.25$ & 0.08 \\
\hline
\end{tabular}

Values are mean $\pm S D, B M D$ : bone mineral density, p: probability, ROM: range of motion, ${ }^{*} \mathrm{p}<0.05$.

significant differences between groups in trunk tilt, and obliquity, as well as hip angles in the sagittal, coronal, and transverse planes. Compared to normal peers, postmenopausal women with low BMD showed a decrease in the trunk rotation $(\mathrm{p}=0.02)$, hip adduction $(\mathrm{p}=0.005)$ and extension moments $(\mathrm{p}=0.008)$. They generated less power at the hip during the early stance $(\mathrm{H} 1 \mathrm{~S})(\mathrm{p}=$ $0.000)$, and swing phase (H3S) $(\mathrm{p}=0.005)$, as well as the

Table 3 Kinetics parameters of the postmenopausal women

\begin{tabular}{|c|c|c|c|}
\hline Variable & $\begin{array}{l}\text { Normal BMD } \\
\text { group }(\mathrm{N}=17)\end{array}$ & $\begin{array}{l}\text { Low BMD } \\
\text { group }(\mathrm{N}=17)\end{array}$ & $\begin{array}{l}\text { MannWhitney } \\
\text { p-value }\end{array}$ \\
\hline \multicolumn{4}{|l|}{ Hip moment } \\
\hline $\begin{array}{l}\text { Adduction moment } \\
\text { (N.m/Kg) }\end{array}$ & $1.01 \pm 0.11$ & $0.88 \pm 0.12$ & $0.005^{* *}$ \\
\hline $\begin{array}{l}\text { Extension moment } \\
(\mathrm{N} . \mathrm{m} / \mathrm{Kg})\end{array}$ & $0.49 \pm 0.22$ & $0.30 \pm 0.12$ & $0.008^{* *}$ \\
\hline $\begin{array}{l}\text { External rotation } \\
\text { moment (N.m/Kg) }\end{array}$ & $0.06 \pm 0.04$ & $0.06 \pm 0.04$ & 0.61 \\
\hline $\begin{array}{l}\text { Internal rotation } \\
\text { moment (N.m/Kg) }\end{array}$ & $0.13 \pm 0.04$ & $0.12 \pm 0.05$ & 0.69 \\
\hline \multicolumn{4}{|l|}{ Hip power } \\
\hline H1S (W/Kg) & $0.57 \pm 0.20$ & $0.31 \pm 0.10$ & $0.000^{* * *}$ \\
\hline H2S (W/Kg) & $0.38 \pm 0.19$ & $0.21 \pm 0.10$ & $0.002^{* *}$ \\
\hline H3S (W/Kg) & $0.71 \pm 0.28$ & $0.44 \pm 0.19$ & $0.005^{* *}$ \\
\hline \multicolumn{4}{|l|}{ Knee power } \\
\hline K1S (W/Kg) & $0.33 \pm 0.09$ & $0.34 \pm 0.13$ & 0.71 \\
\hline K2S (W/Kg) & $0.32 \pm 0.18$ & $0.28 \pm 0.12$ & 0.51 \\
\hline K3S (W/Kg) & $0.67 \pm 0.27$ & $0.52 \pm 0.30$ & 0.18 \\
\hline K4S (W/Kg) & $0.94 \pm 0.37$ & $0.53 \pm 0.27$ & $0.002^{* *}$ \\
\hline \multicolumn{4}{|l|}{ Ankle power } \\
\hline A1S (W/Kg) & $0.28 \pm 0.20$ & $0.21 \pm 0.10$ & 0.31 \\
\hline A2S (W/Kg) & $2.23 \pm 0.44$ & $1.61 \pm 0.46$ & $0.000^{* * *}$ \\
\hline
\end{tabular}

Values are mean $\pm S D, B M D$ : bone mineral density, $\mathrm{H}$ : hip, $\mathrm{K}$ : knee, $\mathrm{A}$ : ankle, S: sagittal, Number: indicates number of power burst, P: probability, ${ }^{*} p<0.05$, ${ }^{* *} \mathrm{p}<0.01,{ }^{* * *} \mathrm{p}<0.001$. 
ankle at the push off $(\mathrm{A} 2 \mathrm{~S})(\mathrm{p}=0.000)$. They absorbed less power at the hip during the stance phase (H2S) $(\mathrm{p}=0.002)$, and the knee during the terminal swing $(\mathrm{K} 4 \mathrm{~S})(\mathrm{p}=0.002)$.

\section{Predictors of BMD}

Table 4 shows Spearman's correlation coefficients between $\mathrm{BMD}$ and gait variables. $\mathrm{L}_{2-4} \mathrm{BMD}$ significantly related to the trunk rotation $(\mathrm{r}=0.38, \mathrm{p}=0.02)$, hip adduction $(\mathrm{r}=0.47, \mathrm{p}=0.005)$, and extension moments $(\mathrm{r}=0.44, \mathrm{p}=0.009), \mathrm{H} 1 \mathrm{~S}(\mathrm{r}=0.55, \mathrm{p}=0.001), \mathrm{H} 2 \mathrm{~S} \quad(\mathrm{r}=$ $0.41, \mathrm{p}=0.017)$, H3S $(\mathrm{r}=0.50, \mathrm{p}=0.003), \mathrm{K} 4 \mathrm{~S}(\mathrm{r}=0.51$, $\mathrm{p}=0.003)$, and A2S $(\mathrm{r}=0.66, \mathrm{p}=0.0001)$. Also, neck BMD significantly related to the trunk rotation $(\mathrm{r}=0.36$, $\mathrm{p}=0.03)$, hip adduction $(\mathrm{r}=0.43, \mathrm{p}=0.011)$ and extension moments $(\mathrm{r}=0.46, \mathrm{p}=0.006)$, H1S $(\mathrm{r}=0.64, \mathrm{p}=0.0003)$, H2S $(r=0.52, p=0.004)$, H3S $(r=0.40, p=0.018), \quad K 4 S$ $(\mathrm{r}=0.47, \mathrm{p}=0.004)$, and A2S $(\mathrm{r}=0.65, \mathrm{p}=0.003)$.

\section{ROC curve analysis}

Figure 1 shows ROC curves of the gait variables related to BMD. Table 5 represents the discriminative properties including AUC, sensitivity, specificity, PPV and NPV. The ability of these variables to discriminate between subjects with or without osteoporosis was $(72 \%, \mathrm{p}=$ $0.016)$ for trunk rotation, $(78 \%, \mathrm{p}=0.0004)$ for hip adduction moment, $(76 \%, \mathrm{p}=0.0013)$ for hip extension moment, $(87 \%, \mathrm{p}<0.0001)$ for H1S, $(79 \%, \mathrm{p}=0.0001)$ for H2S, (77\%, $p=0.0008)$ for H3S, $(81 \%, p=0.0001)$ for $\mathrm{K} 4 \mathrm{~S}$, and $(93 \%, \mathrm{p}<0.0001)$ for A2S. Comparison between AUC of the gait variables showed no significant differences $(P>0.05)$ between them in distinguishing between normal and low BMD women.

\section{Discussion}

This study aimed to provide information about changes in the gait pattern of the postmenopausal women with

Table 4 Spearman's Correlation between BMD and gait variables

\begin{tabular}{llllll}
\hline Variable & \multicolumn{2}{l}{$\mathbf{L}_{\mathbf{2}-\mathbf{4}}$ BMD } & \multicolumn{3}{l}{ Femoral neck BMD } \\
\hline & $r$ & $p$ & $r$ & $p$ \\
Trunk rotation ROM & 0.38 & $0.02^{*}$ & 0.36 & $(0.03)^{*}$ \\
Hip adduction moment (N.m/Kg) & 0.47 & $0.005^{* *}$ & 0.43 & $(0.011)^{*}$ \\
Hip extension moment (N.m/Kg) & 0.44 & $0.009^{* *}$ & 0.46 & $(0.006)^{* *}$ \\
H1S (W/Kg) & 0.55 & $0.001^{* * *}$ & 0.64 & $(0.0003)^{* * *}$ \\
H2S (W/Kg) & 0.41 & $0.017^{*}$ & 0.52 & $(0.004)^{* *}$ \\
H3S (W/Kg) & 0.50 & $0.003^{* *}$ & 0.40 & $(0.018)^{*}$ \\
K4S (W/Kg) & 0.51 & $0.003^{* *}$ & 0.47 & $(0.004)^{* *}$ \\
A2S (W/Kg) & 0.66 & $0.0001^{* * *}$ & 0.65 & $(0.003)^{* *}$ \\
\hline
\end{tabular}

BMD: bone mineral density, ROM: range of motion, $\mathrm{H}$ : hip, $\mathrm{K}$ : knee, $\mathrm{A}$ : ankle, $\mathrm{S}$ : sagittal, Number: indicates number of power burst, r: Spearman's correlation coefficient, P: probability, ${ }^{*} \mathrm{p}<0.05,{ }^{* *} \mathrm{p}<0.01,{ }^{* *} \mathrm{p}<0.001$. low BMD; and to find out which gait parameters related to bone mass. A previous study has already showed a correlation between hip joint moments produced during stepping and hip bone mass in older females [11]. However, this study did not compare between normal and low BMD group or provide information about changes occurred in the trunk, knee, and ankle joints.

In the present study, women with low BMD preserved trunk ROM in the sagittal and coronal planes and hip angles in the three-dimensional planes. However, they showed a decrease in trunk rotation. This finding was in line with Tsauo et al. [17], who reported that spinal motion performance declined, in relation to the severity of BMD, in postmenopausal women without vertebral fracture.

Postmenopausal women, with low BMD, showed a decrease in the external hip extension and adduction moments, power generation at the hip (H1S\&H3S) and ankle (A2S), as well as power absorption at the hip (H2S) and knee (K4S).

Reduced external hip adduction and extension moments agreed with Hurwitz et al. [12], who reported a positive correlation between normalized femoral neck BMD and hip moments in patients with hip osteoarthritis. Reduced hip extension moment at midstance may decrease the $\mathrm{H} 2 \mathrm{~S}$ produced by eccentric contraction of the hip flexors (psoas major and illiacus) [18]. Reduced both variables may affect $\mathrm{BMD}$ at $\mathrm{L}_{2-4}$ and this finding was supported by significant positive correlations found between $\mathrm{L}_{2-4}$ BMD and both hip extension moment and $\mathrm{H} 2 \mathrm{~S}$. The attachment of psoas major along the lateral surfaces of the vertebral bodies of $\mathrm{T}_{12}$ and $\mathrm{L}_{1-5}$ [19] could explain the previous correlations. So, these findings suggest that reduced hip extension moment and associated eccentric hip work may reduce stress on the lumbar region and result in bone loss.

Hip power generation controls the trunk, and collapse of the stance limb; while hip power absorption decelerates the thigh extension [16]. Reduced hip power generation and absorption may represent a problem for women with low BMD. They may not be able to produce a powerful work needed for balance recovery, when large disturbance applied. Postmenopausal women, with low BMD, exhibited decreased knee absorption power produced by eccentric contraction of hamstring [18].

Muscles produce equal amounts of positive and negative work during walking. Muscles contract eccentrically to decelerate the body, and store energy for concentric contraction [16]. During this lengthening behavior, high muscles force is produced [20]. The mode of muscles contraction either concentrically or eccentrically, during rehabilitation of osteoporotic patients, may play a role in maintaining bone mass in the postmenopausal women. However, this needs further 

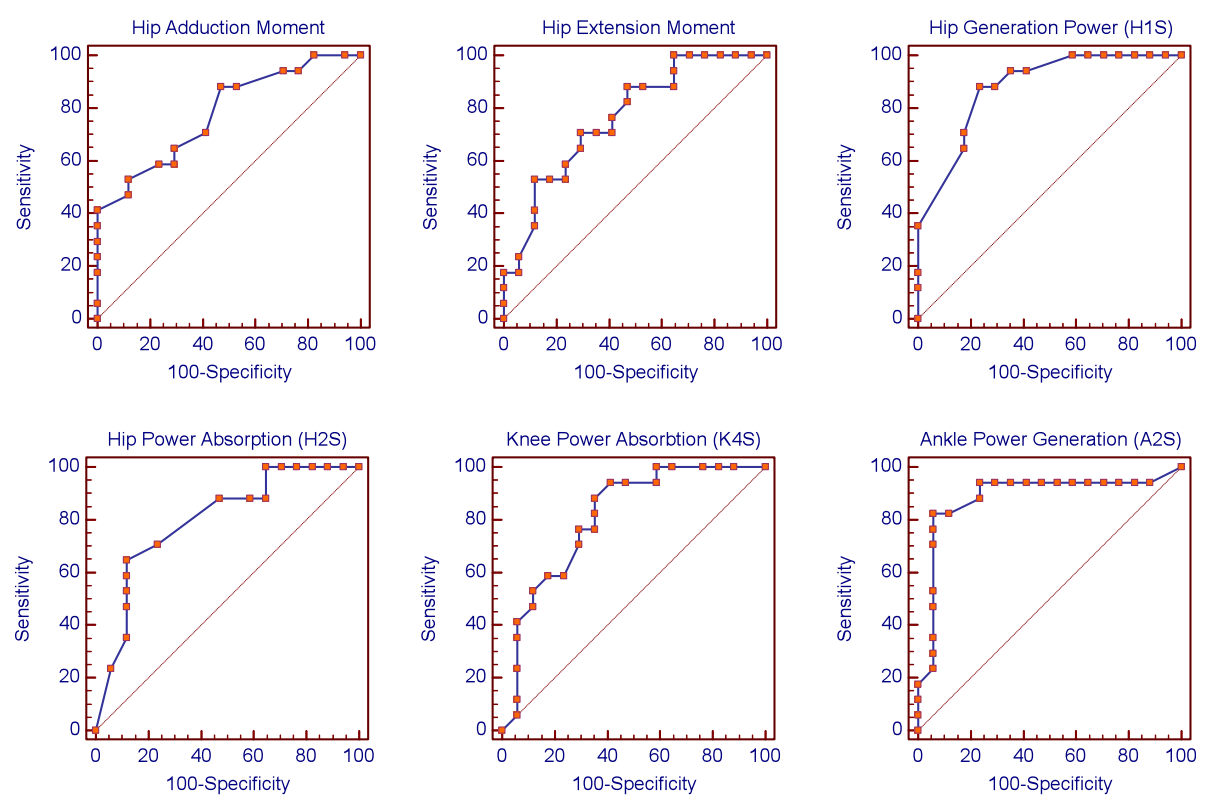

Figure 1 Receiver operating characteristics (ROC) curves for BMD predictors.

electromyography studies to support the previous hypothesis.

The ankle power generation at push-off decreased in low BMD group. Push-off phase is the period during which the muscles power generation is greatest, suggesting that phase is associated with overpowering of the gravitational load [21]. This reflects the importance of this phase in predicting BMD.

Previous studies reported a decreased ankle work in the elderly with limited activities. These studies reported that the elderly might compensate for the reduced ankle work by increasing concentric hip work at early stance [22], or increasing eccentric hip work during midstance [23]. Women with low BMD may not able to compensate for less ankle power due to decreased hip generation and absorption work. Ankle muscle weakness has been suggested as a possible cause for reduced ankle power work in elderly [21].
Decreased hip moments and power generation and absorption in low BMD group are not surprising results because of the strong relationship between muscle strength and bone mass. Muscles induce stains on the skeleton by producing bone bending moments as well as, compressive and tensile force [24]. Sarcopenia and osteoporosis are directly related conditions. Women with osteopenia or osteoporosis showed a greater loss of muscles tissues (sarcopenia) than their normal peers [25]. Sarcopenia results from age-related declines in alpha-motor neurons, sex steroid levels, physical activity [26], and inadequate intake of dietary vitamin $D$ [27]. All of these factors lead to weakness and atrophy of the muscles, which contribute to frailty in elderly [28]. Decline in the activity of the muscles, as a result of weakness, decreases the mechanical stimulation of the muscles on the bones, and aggravates osteoporosis [29].

Table 5 ROC analysis of BMD predictors

\begin{tabular}{|c|c|c|c|c|c|c|}
\hline Variable & AUR $(95 \% \mathrm{Cl})$ & Optimal criterion & Sensitivity $(95 \% \mathrm{Cl})$ & Specificity (95\% Cl) & PPV & NPV \\
\hline Trunk rotation ROM & $0.72(0.54-0.86)$ & $\leq 12.68$ & $52.9(27.8-77)$ & $88.24(63.2-98.5)$ & 81.8 & 65.2 \\
\hline Adduction moment (N.m/Kg) & $0.78(0.60-0.90)$ & $\leq 0.87$ & $41.2(18.4-67.1)$ & $100(80.5-100)$ & 100 & 63 \\
\hline Extension moment (N.m/Kg) & $0.76(0.58-0.89)$ & $\leq 0.34$ & $70.6(44-89.7)$ & $70.6(44-89.7)$ & 70.6 & 70.6 \\
\hline H1S (W/Kg) & $0.87(0.72-0.96)$ & $\leq 0.39$ & $88.2(63.6-98.5)$ & $76.5(50-93.2)$ & 78.9 & 86.7 \\
\hline $\mathrm{H} 2 \mathrm{~S}(\mathrm{~W} / \mathrm{Kg})$ & $0.79(0.62-0.91)$ & $\leq 0.23$ & $64.7(38.3-85.8)$ & $88.2(63.6-98.5)$ & 84.6 & 71.4 \\
\hline H3S (W/Kg) & $0.77(0.60-0.90)$ & $\leq 0.71$ & $100(80.5-100)$ & $52.9(27.8-77)$ & 68 & 100 \\
\hline K4S (W/Kg) & $0.81(0.64-0.92)$ & $\leq 0.82$ & $88.2(63.6-98.5)$ & $64.7(38.8-85.8)$ & 71.4 & 84.6 \\
\hline A2S (W/Kg) & $0.93(0.72-0.99)$ & $\leq 1.78$ & $100(66.4-100)$ & $90(55.5-99.7)$ & 90 & 100 \\
\hline
\end{tabular}

ROC: Receiver operating curve, AUR: area under curve, Cl: confidence interval, PPV: positive predictive value, NPV: negative predictive value, H: hip, K: knee, A: ankle, S: sagittal, Number: indicates number of power burst. 
Work generation and absorption are good indicators to propel and control the lower limbs [16]. Reduced concentric work at the hip and ankle as well as, eccentric work at the hip and knee may suggest that postmenopausal women with low BMD exhibited less propulsion during walking. The previous findings may explain why osteopenic or osteoporotic patients have less stability, and great probability for falls during walking [30,31].

All gait parameters reduced in low BMD group significantly related to $\mathrm{L}_{2-4}$ and femoral neck BMD. Discriminative properties of these gait variables suggest using them as sensitive predictors for low BMD. Seeking for effective interventions, that addresses the affected gait characteristics, is important for maintaining gait mechanics in women with low BMD.

\section{Conclusions}

Postmenopausal women, with low BMD, walk with less hip extension and adduction moments. They produce less power at the hip during early stance and ankle at push off. They absorb less power at the hip during midstance and knee during terminal swing. The previous findings suggest that they had less propulsion and less stability during walking. Hip adduction moment, hip extension moment, H1S, H2S, H3S, K4S and A2S are good markers to predict low $\mathrm{L}_{2-4}$, and femoral neck BMD in the postmenopausal women.

\section{Competing interests}

The authors declare that they have no competing interests.

\section{Authors' contributions}

A. ElDeeb: Protocol/project development, Data collection or management, Data analysis, Manuscript writing/editing; AS. Khodair: Referral of the patients.

\section{Authors' information}

A. EIDeeb is a lecturer of Physical Therapy for Women's Health, Department of Physical Therapy for Women's Health, Faculty of Physical Therapy, Cairo University, Egypt

A. Khodair is a professor of Obstetrics and Gynecology, Department of Obstetrics and Gynecology, Faculty of Medicine, Suez Canal University, Egypt.

\section{Acknowledgment}

The authors thank all postmenopausal women who accepted to engage in this study and all nurses in women's health center, Nasser Institute Hospital for Research and Therapy.

\section{Author details}

'Department of Physical Therapy for Women's Health, Faculty of Physical Therapy, Cairo University, Cairo, Egypt. ²Department of Obstetrics and Gynecology, Faculty of Medicine, Suez Canal University, Suez, Egypt.

Received: 6 January 2014 Accepted: 7 April 2014

Published: 11 April 2014

\section{References}

1. Lane NE: Epidemiology, etiology, and diagnosis of osteoporosis. Am J Obstet Gynecol 2006, 194(2 Suppl):S3-S11.

2. Ahlbor HG, Johnell O, Turner $\mathrm{CH}$, Rannevik G, Karlsson MK: Bone loss and bone size after menopause. N Engl J Med 2003, 349:327-334.

3. Maltais ML, Desroches J, Dionne IJ: Changes in muscle mass and strength after menopause. J Musculoskelet Neuronal Interact 2009, 9:186-197.
4. Auyeung TW, Lee SW, Leung J, Kwok T, Woo J: Age-associated decline of muscle mass, grip strength and gait speed: A 4-year longitudinal study of 3018 community-dwelling older Chinese. Geriatr Gerontol Int 2014, 14(Suppl 1):76-84.

5. Brenneman SK, Barrett-Connor E, Sajjan S, Markson LE, Siris ES: Impact of recent fracture on health-related quality of life in postmenopausal women. J Bone Miner Res 2006, 21:809-816.

6. Adams J, Perry J: Gait analysis: clinical application. In Human Walking 2nd edition. Edited by Rose J, Gamble J. Philadelphia: Williams and Wilkins; 1994:141-164.

7. Lindsey C, Brownbill RA, Bohannon RA, llich JZ: Association of physical performance measures with bone mineral density in postmenopausal women. Arch Phys Med Rehabil 2005, 86:1102-1107.

8. Palombaro KM, Hack LM, Mangione KK, Barr AE, Newton RA, Magri F, Speziale T: Gait variability detects women in early postmenopause with low bone mineral density. Phys Ther 2009, 89:1315-1326

9. Olsson EC: Methods of studying gait. In Gait in rehabilitation. Edited by Smidt GL. New York: Churchill Livingston; 1990:569-597.

10. Bareither ML, Troy KL, Grabiner MD: Bone mineral density of the proximal femur is not related to dynamic joint loading during locomotion in young women. Bone 2006, 38:125-129.

11. Wang MY, Flanagan SP, Song JE, Greendale GA, Salem GJ: Relationships among body weight, joint moments generated during functional activities and hip bone mass in older adults. Clin Biomech (Bristol, Avon) 2006, 21:717-725.

12. Hurwitz DE, Foucher KC, Sumner DR, Andriacchi TP, Rosenberg AG, Galante JO: Hip motion and moments during gait relate directly to proximal femoral bone mineral density in patients with hip osteoarthritis. J Biomech 1998, 31:919-925.

13. Wada M, Maezawa Y, Baba H, Shimada S, Sasaki S, Nose Y: Relationships among bone mineral densities, static alignment and dynamic load in patients with medial compartment knee osteoarthritis. Rheumatology (Oxford) 2001, 40:499-505.

14. Senior, D. Qualisys Medical AB: Qualisys Track Manager User Manual. Gothenburg: NRC Publications Archive (NPArC), 2004.

15. Figueiredo PR, Silva PL, Avelar BS, Chagas PS, Oliveira LC, Mancini MC: Assessment of gait in toddlers with normal motor development and in hemiplegic children with mild motor impairment: a validity study. Braz J Phys Ther 2013, 17:359-366

16. Eng JJ, Winter DA: Kinetic analysis of the lower limbs during walking: what information can be gained from a three-dimensional model? J Biomech 1995, 28:753-758.

17. Tsauo JY, Chien MY, Yang RS: Spinal performance and functional impairment in postmenopausal women with osteoporosis and osteopenia without vertebral fracture. Osteoporos Int 2002, 13:456-460.

18. Whittle MW: Gait analysis: an introduction. 1st edition. Oxford: Bulterworth-Heinemann; 1991

19. Van Dyke JA, Holley HC, Anderson SD: Review of iliopsoas anatomy and pathology. Radiographics 1987, 7:53-84.

20. LaStayo PC, Ewy GA, Pierotti DD, Johns RK, Lindstedt SL: The positive effects of negative work: increased muscle strength and decreased fall risk in a frail elderly population. J Gerontol A Biol Sci Med Sci 2003, 58:M419-M424.

21. Winter DA, Patla AE, Frank JS, Walt SE: Biomechanical walking pattern changes in the fit and healthy elderly. Phys Ther 1990, 70:340-347.

22. Graf A, Judge JO, Ounpuu S, Thelen DG: The effect of walking speed on lower-extremity joint powers among elderly adults who exhibit low physical performance. Arch Phys Med Rehabil 2005, 86:2177-2183.

23. McGibbon CA, Krebs DE: Discriminating age and disability effects in locomotion: neuromuscular adaptations in musculoskeletal pathology. J Appl Physiol 2004, 96:149-160

24. Rittweger J, Beller G, Ehrig J, Jung C, Koch U, Ramolla J, Schmidt F, Newitt D, Majumdar S, Schiessl H, Felsenberg D: Bone-muscle strength indices for the human lower leg. Bone 2000, 27:319-326.

25. Walsh MC, Hunter GR, Livingstone MB: Sarcopenia in premenopausal and postmenopausal women with osteopenia, osteoporosis and normal bone mineral density. Osteoporos Int 2006, 17:61-67.

26. Roubenoff R: Sarcopenia: a major modifiable cause of frailty in the elderly. J Nutr Health Aging 2000, 4:140-142

27. Seo JA, Cho H, Eun CR, Yoo HJ, Kim SG, Choi KM, Baik SH, Choi DS, Park MH, Han C, Kim NH: Association between visceral obesity and sarcopenia and 
vitamin D deficiency in older Koreans: the Ansan Geriatric Study. J Am Geriatr Soc 2012, 60:700-706.

28. Nair KS: Aging muscle. Am J Clin Nutr 2005, 81:953-963.

29. Sinaki M, Fitzpatrick LA, Ritchie CK, Montesano A, Wahner HW: Site-specificity of bone mineral density and muscle strength in women: job-related physical activity. Am J Phys Med Rehabil 1998, 77:470-476.

30. Carter ND, Khan KM, Mallinson A, Janssen PA, Heinonen A, Petit MA, McKay HA, Fall-Free BC Research Group: Knee extension strength is a significant determinant of static and dynamic balance as well as quality of life in older community-dwelling women with osteoporosis. Gerontology 2002, 48:360-368.

31. Liu-Ambrose T, Eng JJ, Khan KM, Carter ND, McKay HA: Older women with osteoporosis have increased postural sway and weaker quadriceps strength than counterparts with normal bone mass: overlooked determinants of fracture risk? J Gerontol A Biol Sci Med Sci 2003, 58:M862-M866.

doi:10.1186/1743-0003-11-55

Cite this article as: ElDeeb and Khodair: Three-dimensional analysis of gait in postmenopausal women with low bone mineral density.

Journal of NeuroEngineering and Rehabilitation 2014 11:55.

\section{Submit your next manuscript to BioMed Central and take full advantage of:}

- Convenient online submission

- Thorough peer review

- No space constraints or color figure charges

- Immediate publication on acceptance

- Inclusion in PubMed, CAS, Scopus and Google Scholar

- Research which is freely available for redistribution 